\title{
Reduced Feedback and Random Beamforming for OFDM MIMO Broadcast Channels
}

\author{
Maralle J. Fakhereddin, Masoud Sharif, and Babak Hassibi
}

\begin{abstract}
It has been shown that random beamforming using partial channel state information (CSI) achieves the same throughput scaling as obtained from dirty paper coding for a broadcast (downlink) channel with $M$ transmit antennas and $K$ users where $K$ is large [1]. In this paper, we apply this scheme to wideband MIMO broadcast channels. By using OFDM, an $L$-tap wideband channel can be decomposed to $N$ parallel narrowband channels (subcarriers), where $N>L$. Neighboring subcarriers are highly correlated. Therefore, we consider neighboring subcarriers as a cluster and find the closed form solution for the joint characteristic function of SINR values at two subcarriers in a cluster. We show numerically how the knowledge of the quality of the center subcarrier sheds light about the quality of other subcarriers in the same cluster, and address the issue of cluster size. In addition, through complex and asymptotic analysis, we show that for cluster size of order $\frac{N}{L \sqrt{\log K}}$ (for large $K$ ), users need only feedback the best SINR at the center subcarrier of each cluster in order for the transmitter to perform opportunistic beamforming and maintain the same throughput scaling as when full CSI is available. Using simulation results, we verify our analytical result and show that even fewer feedback can be tolerated, and larger clusters $\left(\frac{N}{2 L}\right)$ can be implemented for a small throughput hit.
\end{abstract}

Index Terms-Channel state information, OFDM, broadcast channel, random beamforming, multi-user diversity, wireless communications.

\section{INTRODUCTION}

$\mathbf{T}$ HERE has been growing interest in the study of the capacity region of Gaussian multiple-input multiple-output (MIMO) broadcast channels [2], [3], [4] . Recently, it has been shown that dirty paper coding (DPC) achieves the capacity region of the Gaussian MIMO broadcast channel [5]. This scheme assumes perfect channel state information (CSI) at the transmitter, and achieves throughput that scales linearly with the number of transmit antennas [6]. However, full channel knowledge is not always attainable or practical, especially if the channel is varying rapidly or in systems with many users. As a result, there has been increased interest in studying the effect of reduced CSI on multi-user diversity and sum rate

Paper approved by S. N. Batalama, the Editor for Spread Spectrum and Estimation of the IEEE Communications Society. Manuscript received July 17, 2006; revised June 15, 2007, January 19, 2008, and June 5, 2008.

This work was supported in part by the National Science Foundation under grant no. CCR-0133818, by the office of Naval Research under grant no. N00014-02-1-0578, and by Caltech's Lee Center for Advanced Networking. A conference version of this paper was presented at SPAWC, June 2005.

B. Hassibi is with Department of Electrical Engineering, California Institute of Technology, Pasadena, CA 91125 (e-mail: hassibi@systems.caltech.edu).

M. Sharif is with the Department of Electrical and Computer Engineering, Boston University, Boston, MA 02215 (e-mail: sharif@bu.edu).

M. Fakhereddin is with VMware, Inc. Palo Alto, CA 94304 (e-mail: maralle@systems.caltech.edu).

Digital Object Identifier 10.1109/TCOMM.2009.12.060236 capacity, for example [1], [7], [8], [9], [10], [11], [12] among others. It was suggested in [1] that, using $M$ random beams and partial feedback, opportunistic beamforming is performed at the transmitter. Each user (receiver) calculates and feeds back its best signal-to-noise-plus-interference ratio (SINR) and the beam index at which this maximum occurs. The transmitter then chooses the user with the highest SINR for each beam and transmits its information. This scheme requires less feedback and is computationally feasible, still it achieves the linear scaling in throughput that is achieved with DPC. This has been shown for narrowband broadcast channels with a fixed number of transmit antennas and large number of users.

We investigate the generalization of this scheme to wideband broadcast channels (with independent and identically distributed (i.i.d.) channel taps). Wideband channels are desireable due to the increased need to drive bit rates higher. Using orthogonal frequency division multiplexing (OFDM), an $L$-tap wideband channel can be decomposed to $N$ parallel subcarriers, where $N>L$. As a result, neighboring subcarriers are highly correlated, and therefore have similar channel quality (with high probability). Recently, it has been suggested in [7] that neighboring subcarriers are grouped in a cluster. Then each user need only feedback the signal-to-noise ratio (SNR) values and indices of its strongest clusters. Based on this reduced feedback, the transmitter sends one beam to the user with the highest SNR per cluster. Using simulation results, [7] shows that this scheme can be implemented without significantly sacrificing performance. It has also been suggested in [10] that one bit feedback can be used at each subcarrier in order to perform opportunistic subcarrier allocation. In [12], dynamic allocation alogorithms were investigated for multiple antenna wideband systems to show that multi-user diversity can still be achieved even under a hard fairness constraint.

In this paper, we closely investigate the correlation between neighboring subcarriers in order to minimize the amount of CSI needed at the transmitter. We first find the closed form solution for the joint characteristic function of SINR values at two subcarriers in a cluster. Through numerical results, we address the issue of how many neighboring subcarriers should be grouped together in a cluster so that we need only feedback one value that is useful to the transmitter for throughput maximization. In fact, we suggest that each user need only feedback its best SINR value at the center subcarrier of each cluster as long as the edge subcarrier is within some distance (that we find numerically) from the center of the cluster. The transmitter then performs opportunistic beamforming (by constructing $M$ random beams rather than a single beam). Next, we look into the effect of this partial feedback on the throughput. More specifically, we want to 
know if we are able to maintain the same throughput scaling as when full CSI is available. Through complex and asymptotic analysis, we prove that for a wideband broadcast channel with $M$ transmit antennas, $K$ single-antenna users (where $K$ is large) and cluster size of order $\frac{N}{L \sqrt{\log K}}$, we achieve the same throughput scaling as when full CSI is available at the transmitter, however with less complexity and feedback.

Monte Carlo simulations verify our analytical results and show that larger cluster size $\left(\frac{N}{2 L}\right)$ can also be implemented for a small throughput hit. We also investigate variations to our scheme that require even fewer feedback and can achieve high throughput results. First, we introduce a threshold that each user should compare the SINR value they intend to feed back, and only send those that are larger than or equal to this threshold. This thresholding idea was first suggested by [9], [13]. Another variation requires users to only send 1 bit ('1') for each SINR value that is higher than or equal to the set threshold. This idea was investigated by [10], [11] for single-antenna OFDM systems. We also compare our proposed scheme to a scheme that performs linear interpolation based on feeding back two SINR values around the center. Linear interpolation has been investigated for channel estimation in OFDM systems [14], [15].

The remainder of this paper is as follows. Section 2 describes the channel model including feedback and scheduling schemes. Section 3 states the problem. Our analysis begins in section 4 where we find the closed form solution for the joint characteristic function of neighboring subcarriers, and present our numerical results. Section 5 includes the throughput scaling and Monte Carlo simulation results. Section 6 concludes the paper. Details of complex and asymptotic analysis used in the throughput result are listed in the appendices.

\section{Channel Model}

We consider an $L$-tap wideband broadcast channel with $M$-antenna transmitter and $K$ single-antenna receivers. The number of channel taps $L$ is dictated by the delay spread. In practice, wideband channels can be represented by 4 or 5 channel taps [16]. We assume the channel taps are independent, complex Gaussian random variables. We form an $L \times M$ channel matrix $H$ which consists of zero-mean unit variance circularly symmetric complex Gaussian random variables. We denote the $m^{\text {th }}$ column vector of $H$ by $H_{l}^{m}$ where $H_{l}^{m}=\left[\begin{array}{llll}h_{0}^{m} & h_{1}^{m} & \ldots & h_{L-1}^{m}\end{array}\right]^{T}$.

We start with a discrete-time baseband channel model. At time $t$ and sampling at multiples of $\frac{1}{W}$, the input-output relationship between any of the transmit antennas and any user is

$$
y_{m}[t]=\sum_{l=0}^{L-1} h_{l, m} x[t-l]+w[t]
$$

where $y_{m}$ is the sampled output at time $t$ from the $m^{\text {th }}$ transmit antenna, $x$ is the sampled input transmitted at time $t-l$ and $w$ is additive white Gaussian noise.

Using OFDM, data symbols modulate $N$ subcarriers separated by $\frac{W}{N}$. The discrete Fourier transform of the $L$-tap chan- nel gives the frequency response at subcarrier $q$ as follows,

$$
H_{m}(q)=\sum_{l=0}^{L-1} h_{l, m} e^{-j 2 \pi q \frac{l W}{N}}, \quad q=1, \ldots, N .
$$

In matrix form, (2) can be written as $H_{m}(q)=V_{q} H_{m}$ where $V_{q}=\left[\begin{array}{llll}1 & e^{-j 2 \pi q \frac{W}{N}} & \ldots & e^{-j 2 \pi q \frac{W(L-1)}{N}}\end{array}\right]$ and $H_{m}$ represents the channel at the $m^{\text {th }}$ beam as explained in the next section. As a result of decomposing the $L$-tap channel into $N$ parallel subcarriers where $N>L$, neighboring subcarriers are highly correlated. The autocorrelation function depends solely on the frequency separation between subcarriers. Since the width of each subcarrier is $\frac{W}{N}$, it is straightforward to show that there are roughly $\frac{N}{2 L}$ neighboring subcarriers that are highly correlated [16]. We further quantify this correlation by finding the joint characterization function of neighboring subcarriers in section 4. Correlated subcarriers are grouped in a cluster [7], and we investigate the cluster size and a good channel quality indicator for the whole cluster. Using this partial feedback, we adopt a transmission scheme on each subcarrier that maximizes the throughput [1] as explained below.

\section{A. Signaling Scheme}

We construct $M$ random orthonormal beams $\phi_{m}(M \times 1)$ for $m=1, \ldots, M$. At time $t$, the $m^{\text {th }}$ vector is multiplied by the transmit symbol $s_{m}^{q}$. Note that we use the same beams for the entire cluster, not each subcarrier. The baseband envelope of the transmitted signal on subcarrier $q$ is $S_{q}(t)=\sum_{m=1}^{M} s_{m}^{q}(t) \phi_{m}(t)^{1}$. The channel gains at the $m^{\text {th }}$ beam are represented by $L \times 1$ vector $H_{m}$ where $H_{m}=H \phi_{m}$. Note that $H_{m}$ maintains the same distribution as $H$ with variance $M I_{L}$.

We assume that the $k^{\text {th }}$ receiver has full knowledge of $H_{m}^{k}$ for $m=1, \ldots, M$ which can be readily available through training. We consider a homogeneous network which means that all users have the same received SNR. Therefore, the $k^{\text {th }}$ receiver is able to compute the following $M \operatorname{SINR}_{q, m}^{k}$ values by assuming that the signal $s_{m}$ is its desired signal and the other $s_{i}$ signals are interference as follows,

$\mathrm{SINR}_{q, m}^{k}=\frac{H_{m}^{k^{*}} V_{q}^{*} V_{q} H_{m}^{k}}{\frac{1}{\rho}+\sum_{i=1, i \neq m}^{M} H_{i}^{k^{*}} V_{q}^{*} V_{q} H_{i}^{k}}, m=1, \ldots, M$

where $\rho$ is the SNR for all users and $q$ is the index of the subcarrier. We will refer to the subcarrier at the center by the index $q$ from this point on.

\section{B. Feedback Scheme}

We propose that each user feeds back the best SINR value at the center subcarrier of each cluster (in addition to corresponding beam index). This feedback can be expressed as $\max _{1 \leq m \leq M} \operatorname{SINR}_{q, m}^{k}$. The transmitter then assigns the $m^{t h}$ beam to the user with the highest SINR; $\max _{1 \leq k \leq K} \mathrm{SINR}_{q, m}^{k}$. The throughput of each subcarrier is estimated by $R_{q}=$

\footnotetext{
${ }^{1}$ It is worth mentioning that the IFFT is applied after the beams are constructed. In other words, the beamforming is applied in the frequency domain. This enables transmitting to multiple receivers at the same time.
} 
$E\left\{\sum_{m=1}^{M} \log \left(1+\max _{1 \leq k \leq K} \operatorname{SINR}_{q, m}^{k}\right)\right\}$, and the throughput of the system is $R=\sum_{q=1}^{N} R_{q}$, where $N$ is dictated by the transmission bandwidth. Note that for $K \rightarrow \infty$, this is a tight estimate [1]. ${ }^{2}$

Our proposed scheme requires each user to feed back one value per cluster. In order to further reduce the amount of feedback, we assume that $\operatorname{SINR}_{q, m}^{k} \geq \gamma$ where $\gamma$ is a specified threshold of order $\log K^{3}$. We are able to set this threshold since we know that $\max _{1 \leq k \leq K} \operatorname{SINR}_{q, m}^{k}$ behaves like $(\log K)$ with high probability [1]. The amount of feedback from each user then reduces to the number of clusters $\times \operatorname{Pr}\left(\operatorname{SINR}_{q} \geq \gamma\right)$. We observe that there is a tradeoff between the amount of feedback sent (or equivalently the size/number of clusters) and the throughput. In the following section, we further discuss this tradeoff and state our problem.

\section{Problem Statement}

In this paper, we tackle two main questions. The first question addresses how big clusters should be (and subsequently how much feedback should we have) so that the condition of the center subcarrier is indeed a valid representative of the condition of (most of) the subcarriers in the cluster. The second question concerns the throughput scaling of the wideband MIMO broadcast channel when only partial CSI is available at the transmitter.

The size of the cluster influences how correlated the subcarriers are. Small cluster sizes guarantee that the subcarriers are highly correlated and that their channel quality is almost the same, therefore resulting in higher throughput (at the expense of more feedback). Whereas wider cluster sizes reduces the amount of feedback but will include less correlated subcarriers in the cluster (especially toward the edges of the cluster), which will subsequently decrease the throughput. We show that channel quality at the center subcarrier is a valid representative (and therefore sufficient feedback) for the quality of the channels at (most of) subcarriers in a cluster when the cluster size is within a specific value that we find numerically.

In fact, since we know that the best SINR over all users behaves like $\rho \log K$, we are able to identify that we are interested in SINR values of order $\log K$. As the cluster size increases, the likelihood of the edge subcarrier (the farthest from the center) being of this order decreases. We show through complex and asymptotic analysis that if the size of the cluster is of order $\frac{N}{L \sqrt{\log K}}$, and if the center subcarrier of this cluster has SINR of order $\log K$, then with high probability the SINR at the edge subcarrier is also of order $\log K$. In other words, we show that the following probability,

$$
\operatorname{Pr}\left\{\operatorname{SINR}_{p, m}^{k} \geq \gamma_{p} \mid \operatorname{SINR}_{q, m}^{k} \geq \gamma_{q}\right\}
$$

is close to one when $p-q=\frac{N}{L \sqrt{\log K}}^{4}, M$ fixed, $K$ increasing,

\footnotetext{
${ }^{2}$ Note that the transmitter should transmit to the neighboring subcarriers of that cluster after backing off some specified value in order to avoid outage. However we do not consider this backoff in this paper.

${ }^{3}$ Having identically and independently distributed users implies that all SINR's have the same distribution and are independent across users. This allows us to set the aforementioned threshold for all users as shown in Section 3.

${ }^{4}$ In our analysis, we equivalently assume that the correlation coefficient of $p$ and $q$ is $1-o\left(\frac{1}{\log K}\right)$.
}

$\gamma_{q}=\alpha_{q} \log K, \gamma_{p}=\alpha_{p} \log K$ where $\alpha_{q}$ and $\alpha_{p}$ are constants and $\alpha_{q}>\alpha_{p}$. In eq. (4), the user index $k$ corresponds to the user with the highest center SINR for the studied cluster among all $K$ users. This "best" user can be different for different clusters. The details of analysis are provided in the following two sections and appendices.

\section{Evaluating Conditional Probability}

We substitute eq. (3) into eq. (4), and denote $\mathcal{P}=$ $H_{m}^{k^{*}} V_{p}^{*} V_{p} H_{m}^{k}-\gamma_{p} \sum_{i=1, i \neq m}^{M} H_{i}^{k^{*}} V_{p}^{*} V_{p} H_{i}^{k}-\frac{\gamma_{p}}{\rho}$ and $\mathcal{Q}=$ $H_{m}^{k^{*}} V_{q}^{*} V_{q} H_{m}^{k}-\gamma_{q} \sum_{i=1, i \neq m}^{M} H_{i}^{k^{*}} V_{q}^{*} V_{q} H_{i}^{k}-\frac{\gamma_{q}}{\rho}$.

Eq. (4) can be rewritten as

$$
\begin{aligned}
& \operatorname{Pr}\left\{\operatorname{SINR}_{p, m}^{k} \geq \gamma_{p} \mid \operatorname{SINR}_{q, m}^{k} \geq \gamma_{q}\right\} \\
& =\operatorname{Pr}\{\mathcal{P} \geq 0 \mid \mathcal{Q} \geq 0\}=\frac{\operatorname{Pr}\{\mathcal{P} \geq 0, \mathcal{Q} \geq 0\}}{\operatorname{Pr}\{\mathcal{Q} \geq 0\}} .
\end{aligned}
$$

To calculate (5), we need to find the joint probability of $\mathcal{P}$ and $\mathcal{Q}$. Since $\mathcal{P}$ and $\mathcal{Q}$ are correlated, this joint probability should be evaluated. Using the Fourier Transform representation of the unit function, $U(x)=\frac{1}{2 \pi} \int_{-\infty}^{\infty} e^{j w x}\left(\frac{1}{j w}+\pi \delta(w)\right) d w$, this joint probability can be written as,

$$
\begin{aligned}
\operatorname{Pr}\{\mathcal{Q} \geq 0, \mathcal{P} \geq 0\} & =\frac{1}{4}+\frac{1}{4 \pi} \int \frac{\Phi(\mathcal{P})}{j w_{p}} d w_{p} \\
& +\frac{1}{4 \pi} \int \frac{\Phi(\mathcal{Q})}{j w_{q}} d w_{q} \\
& +\frac{1}{4 \pi^{2}} \iint \frac{\Phi(\mathcal{P}, \mathcal{Q})}{j w_{p} j w_{q}} d w_{p} d w_{q}
\end{aligned}
$$

where $\Phi$ stands for the characteristic function and the integration limits are from $-\infty$ to $\infty$.

Note that the marginal terms (second and third terms in eq. (6)) can be easily evaluated from the marginal distribution of SINR [1]. We can show that $\frac{1}{4 \pi} \int \frac{\Phi(\mathcal{Q})}{j w_{q}} d w_{q}=$ $-\frac{1}{4}-\frac{1}{2} \frac{e^{-\frac{\gamma_{q}}{\rho L M}}}{\left(1+\gamma_{q}\right)^{M-1}}$ and $\frac{1}{4 \pi} \int \frac{\Phi(\mathcal{P})}{j w_{p}} d w_{p}=-\frac{1}{4}-\frac{1}{2} \frac{e^{-\frac{\gamma_{p}}{\rho L M}}}{\left(1+\gamma_{p}\right)^{M-1}}$. However, these terms cancel out with terms that appear during the evaluation of the double integral in the fourth term of eq. (6). Therefore, the main analysis of the joint probability lies in evaluating the joint characteristic funtion and the double integral in eq. (6). Next, we find the closed form for the joint characteristic function $\Phi(\mathcal{P}, \mathcal{Q})$.

\section{A. Joint Characteristic Function of Neighboring subcarriers}

The joint characteristic function of $\mathcal{P}$ and $\mathcal{Q}, \Phi(\mathcal{P}, \mathcal{Q})=$ $E\left(e^{j w_{p} \mathcal{P}+j w_{q} \mathcal{Q}}\right)$. We assume that the transmit antennas are far enough to assume independent channels.

Therefore we can write (7) (on the next page). Using properties of joint pdf of Gaussian random variables [17], the closed form solution for the joint characteristic function can be found as shown in (8) (on the next page), where $f=\frac{\sin ^{2}\left(\pi(p-q) \frac{L}{N}\right)}{\sin ^{2}\left(\pi(p-q) \frac{1}{N}\right)}$. Note that when $p-q=\frac{N}{L}, f=0$ which results in $\mathcal{P}$ and $\mathcal{Q}$ being independent. Also if we assume that the cluster size is 1 subcarrier, and therefore $p=q$, then $\left(L^{2}-f\right)=0$, and the joint characteristic function reduces to a marginal function. 


$$
\begin{aligned}
& \Phi(\mathcal{P}, \mathcal{Q})=e^{\frac{-1}{\rho}\left(j w_{p} \gamma_{p}+j w_{q} \gamma_{q}\right)} \int_{-\infty}^{\infty} \ldots \int_{-\infty}^{\infty} e^{-\sum_{i=1, i \neq m}^{M} H_{i}^{k^{*}}\left(j w_{p} \gamma_{p} V_{p}^{*} V_{p}+j w_{q} \gamma_{q} V_{q}^{*} V_{q}\right) H_{i}^{k}} \underbrace{p\left(H_{1}^{k}\right) \ldots p\left(H_{M}^{k}\right)}_{M-1 \text { terms }} d H_{1}^{k} \ldots d H_{M}^{k} \\
& \times \int_{-\infty}^{\infty} e^{H_{m}^{k^{*}}\left(j w_{p} V_{p}^{*} V_{p}+j w_{q} V_{q}^{*} V_{q}\right) H_{m}^{k}} p\left(H_{m}^{k}\right) d H_{m}^{k} \\
& \Phi(\mathcal{P}, \mathcal{Q})=\frac{e^{\frac{-1}{\rho}\left(j w_{p} \gamma_{p}+j w_{q} \gamma_{q}\right)}}{M^{L M} \quad \operatorname{det}\left[\frac{I_{L}}{M}-\left(j w_{p} V_{p}^{*} V_{p}+j w_{q} V_{q}^{*} V_{q}\right)\right] \quad \operatorname{det}\left[\frac{I_{L}}{M}+\left(j w_{p} \gamma_{p} V_{p}^{*} V_{p}+j w_{q} \gamma_{q} V_{q}^{*} V_{q}\right)\right]^{M-1}} \\
& =\frac{e^{\frac{-1}{\rho}\left(j w_{p} \gamma_{p}+j w_{q} \gamma_{q}\right)}}{\left[1-\left(j w_{p}+j w_{q}\right) L M-w_{p} w_{q}\left(L^{2}-f\right) M^{2}\right]\left[1+\left(j w_{p} \gamma_{p}+j w_{q} \gamma_{q}\right) L M-w_{p} w_{q} \gamma_{p} \gamma_{q}\left(L^{2}-f\right) M^{2}\right]^{M-1}}
\end{aligned}
$$

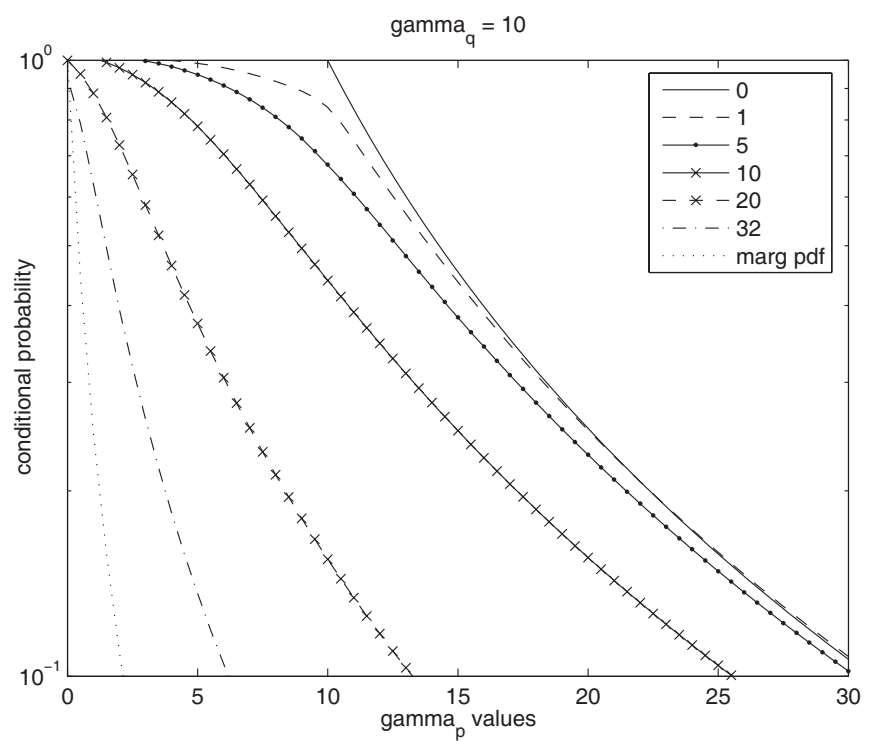

Fig. 1. Numerical Result for the conditional probability of the edge SINR $\geq \gamma_{p}$ if center SINR $\geq \gamma_{q}$ for various $p-q$ distances.

\section{B. Numerical Analysis - Cluster Size}

From the closed form of the joint characteristic function in eq. (8) along with the knowledge of the marginal distribution of the SINR [1], we can find the conditional probability in eq. (4) numerically. Fig. 1 plots the value of the probability that the edge $\operatorname{SINR}_{\mathrm{p}} \geq \gamma_{\mathrm{p}}$ conditional on the fact that the center $\operatorname{SINR}_{\mathrm{q}} \geq \gamma_{\mathrm{q}}$ for various $p-q$ distances for a wideband broadcast channel with a 3 -antenna transmitter, single-antenna users, and 256 subcarriers. We observe that when we set $\gamma_{q}=$ 10 and when $p-q=0$ (which means that there is only one subcarrier in the cluster), we know with probability $=1$ that $\gamma_{p}$ is as high as $\gamma_{q}$. This probability becomes 0.7 when $p-q=5$, and decreases to 0.5 as the distance increases to 10 . Finally at $p-q=32$, the conditional probability becomes very close to the marginal probability indicating that $p$ and $q$ are so far that they become uncorrelated. In this case, the knowledge of the channel quality at the center does not shed light about the quality at the edge of the cluster. This numerical result is very helpful in deciding what the cluster size should be for any value of the conditional probability. For example, if one knows that the center SINR behaves within some threshold and aims to have the edge SINR also within the same threshold with probability 0.75 , then the cluster size should be kept to 7 or 8 subcarriers.

\section{Throughrut Scaling Analysis}

In this section, we answer the second question which addresses the throughput scaling of the wideband MIMO broadcast channel when only partial feedback is available at the transmitter. Through complex and asymptotic analysis (detailed in appendices A and B), the joint characteristic function (8) enables us to find the joint probability distribution as,

$$
\operatorname{Pr}\{\mathcal{P} \geq 0, \mathcal{Q} \geq 0\}=\frac{e^{-\frac{\gamma_{q}}{\rho M L}}}{\left(1+\gamma_{q}\right)^{M-1}}[1-o(1)]^{M-1} .
$$

Since we know the marginal distribution of SINR, the denominator in (5) results in $\frac{e^{-\frac{\gamma_{q}}{\rho M L}}}{\left(1+\gamma_{q}\right)^{M-1}}$ [1]. Our final result for the conditional probability becomes,

$$
\operatorname{Pr}\left\{\operatorname{SINR}_{p, m}^{k} \geq \gamma_{p} \mid \operatorname{SINR}_{q, m}^{k} \geq \gamma_{q}\right\}=1-o(1)
$$

when $p-q=\frac{N}{L \sqrt{\log K}}, M$ is fixed and $K$ increasing, $\gamma_{q}=\alpha_{q} \log K, \gamma_{p}=\alpha_{p} \log K$ and $\alpha_{q}>\alpha_{p}$. For the user with the highest center SINR, this proves that when SINR at the center subcarrier is of order $\log K$, and if we keep our cluster size small enough so that $p-q=\frac{N}{L \sqrt{\log K}}^{5}$, then we can say with high probability that neighboring subcarriers are highly correlated. For this reason, SINR at the edge subcarrier is also of order $\log K$, where $K$ is large. As a result, we can say that all subcarriers chosen for transmission have SINRs of order $\log K$, and consequently throughput scaling is $M N \log \log K$ where $N$ is the number of subcarriers and dictated by the transmission bandwidth. This is the same throughput scaling as when the transmitter has full information about the channel at each subcarrier, yet with much fewer feedback and less computation complexity. In fact, feedback can be further reduced to $L \sqrt{\log K} \times \operatorname{Pr}\left(\operatorname{SINR}_{\mathrm{q}, \mathrm{m}} \geq \gamma\right)$ per user as previously explained in section II.B. Next, we verify our analytical results using Monte Carlo simulations.

\footnotetext{
${ }^{5}$ It is not easy to perform the analysis for any cluster size since $L^{2}-f$ becomes a constant and it is then difficult to obtain the roots of the polynomial which is raised to power $(M-1)$ in Eq. (A.2)
} 


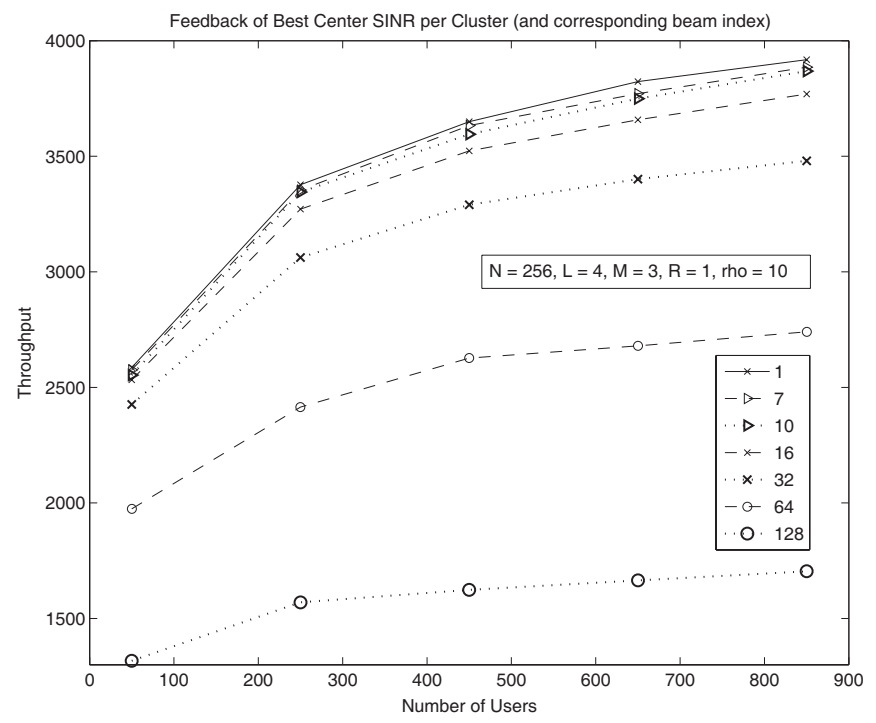

Fig. 2. Throughput vs. Number of users for different cluster sizes. In this scheme, each user feeds back its best center SINR per cluster (along with the corresponding beam index).

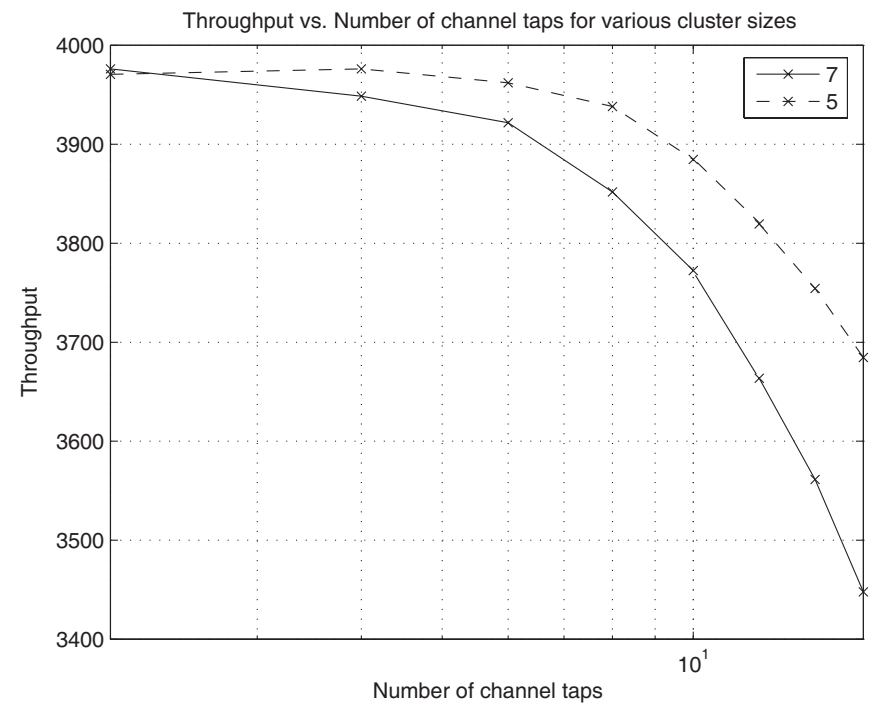

Fig. 3. Throughput vs. Number of channel taps for different cluster sizes $(\mathrm{N}=256, \mathrm{M}=3, \mathrm{SNR}=10, \mathrm{~K}=1000)$.

\section{A. Simulation Results}

To verify our results, we conduct Monte Carlo simulations for a wideband broadcast channel with a 3-antenna transmitter, single-antenna users, and 256 subcarriers. Fig. 2 displays the throughput as a function of number of users for various cluster sizes, and shows that cluster size $\frac{N}{2 L}=32$ results in a very small throughput loss when compared with the throughput that results from cluster size of 1 subcarrier (where the transmitter has full information at each subcarrier). However, as suggested by our analysis, Fig. 2 also indicates that smaller cluster sizes (7, 10 and even 16) result in throughput scaling as with full CSI. This demonstrates that when we stay in the region of the highest SINR values (those that behave like $\log K)$ and with $L^{2}-f \rightarrow 0$, we preserve the throughput scaling as with full CSI. Note that larger cluster sizes (i.e.

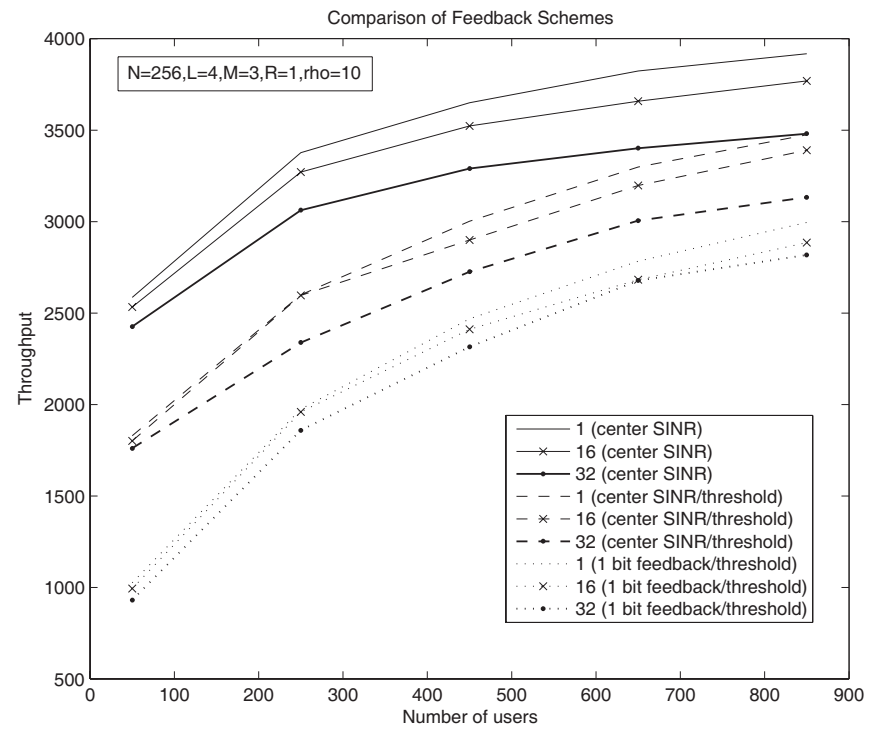

Fig. 4. Throughput vs. Number of users for different feedback schemes and cluster sizes. Solid lines for feeding back the best center SINR per cluster. Dashed lines for feeding back the best center SINR per cluster provided that it is $\geq$ threshold $(3 \log K)$. Dotted lines for feeding back 1 bit for each cluster where the best center SINR is $\geq$ threshold $(3 \log K)$

64 and 128) result in significantly lower throughput. In Fig. 3 , we plot the throughput versus the number of channel taps $L$ for cluster sizes 5 and 7 . This figure indicates how much variation (spread) the channel is able to withstand (for a certain cluster size) without too much throughput reduction. Another interesting observation from Fig. 3 is that we can treat a small number of subcarriers as a single subcarrier, and still maintain flat channel response.

To further reduce feedback (especially when the cluster size is small), we further assume that $\operatorname{SINR}_{q, m}^{k} \geq \gamma$ where $\gamma$ is a specified threshold of order $\log K$ as previously discussed in section 2.2. We can set this threshold since we know that $\max _{1<k \leq K} \operatorname{SINR}_{q, m}^{k}$ behaves like $\rho \log K$ with high probability. We set $\rho=10$ and $\gamma=3 \log K$ in Fig. 4 , and observe only a small throughput hit (about $300 \mathrm{bits} / \mathrm{sec}$ for cluster sizes 16 and 32 and large number of users). The optimal threshold value is an interesting problem which we do not investigate in this paper, but has been discussed in [11]. To reduce feedback even more, we follow a similar scheme as that suggested in [10], and request that users with SINR $\geq \gamma$ send only 1 bit ' 1 ' indicating that their channel is good. However, in this case the transmitter cannot identify which user has the best SINR over each beam, and will therefore randomly choose any of the users who fed back ' 1 ' for that beam. In Fig. 4, the effect of this scheme is observed by another hit in throughput (about $500 \mathrm{bits} / \mathrm{sec}$ for cluster sizes 1 and 16, and about $400 \mathrm{bits} / \mathrm{sec}$ reduction for cluster size 32 at large number of users). This is due to the transmitter not optimizing over the best SINR. Also note that the gap between cluster sizes 1,16 and 32 decreases.

For large clusters, the center SINR is not a good indicator for the edge SINR. However, the center is still a good representative of its neighboring channels. For this reason, we investigate through simulations the effect of each user feeding back two SINR values (around the center) per cluster for large 


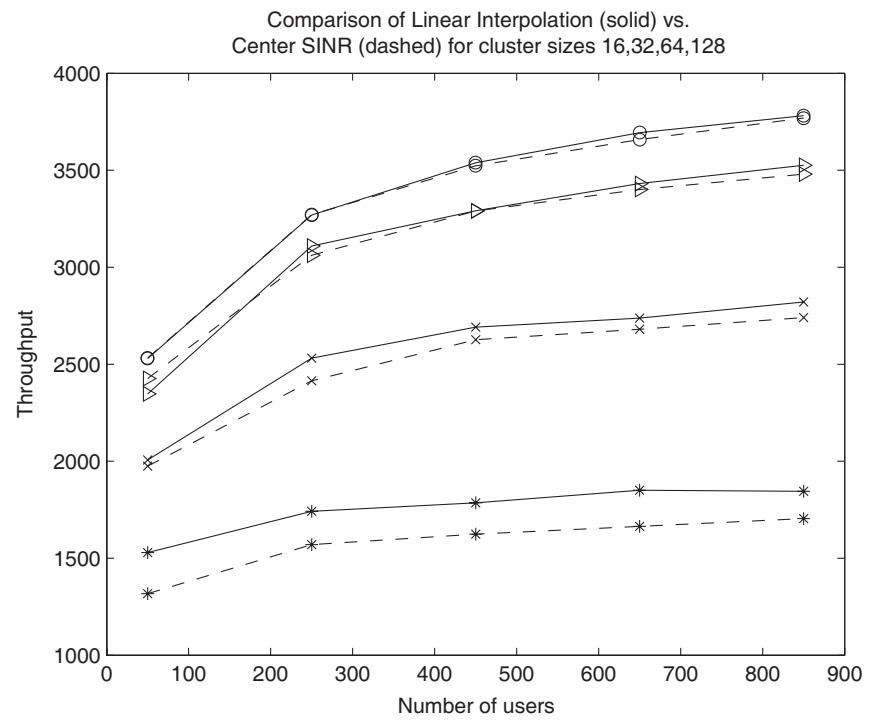

Fig. 5. Throughput vs. Number of users for different cluster sizes $(16,32,64,128)$ comparing between linear interpolation (solid lines) and the proposed scheme of feeding back best center SINR per cluster (dashed lines).
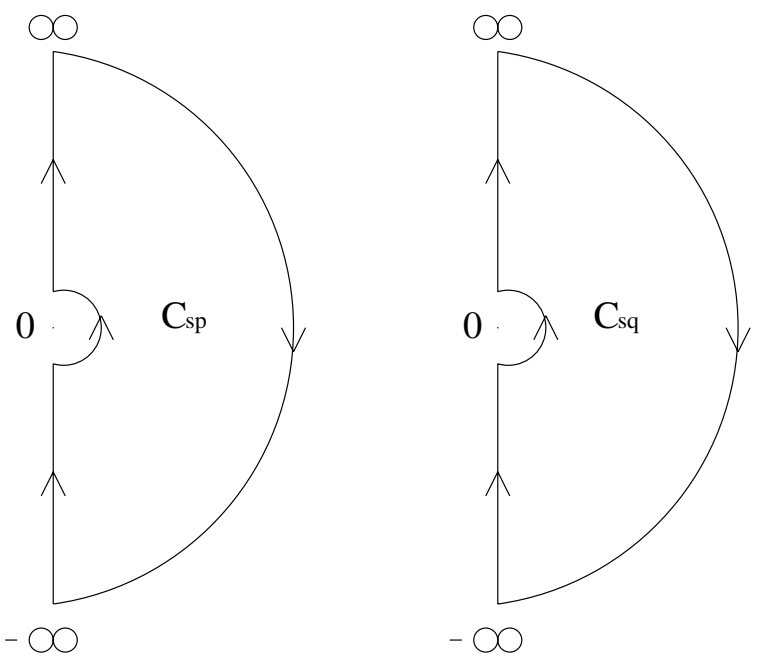

Fig. 6. Illustration of contours $C_{s_{p}}$ and $C_{s_{q}}$.

cluster sizes ( $\frac{N}{L}$ and larger). This way, linear interpolation can be performed at the transmitter to obtain all SINR values between the two fed back, and the average SINR can be calculated. This leads to better performance results for larger clusters. Fig. 5 illustrates that this scheme does as well as our proposed scheme for small clusters, and therefore there is no added benefit to the extra feedback. As expected, when clusters are large, linear interpolation results in slightly better throughput (about $150 \mathrm{bits} / \mathrm{sec}$ for cluster size 128) with the added price of feeding back two values per cluster rather than one.

\section{CONCLUSIONS}

In this paper we answer two questions. We first find the closed form solution for the joint characteristic function of SINR values of two neighboring subcarriers, and find numerically the conditional probability of the edge SINR being as good as the center SINR. This numerical result tells us how many subcarriers we can group together in a clusterso that with some probability (that we specify) the performance at the edge of the cluster is almost as that of the center. This is important since then we can reduce the feedback to only one value per cluster. Next we find the throughput of wideband multiantenna broadcast channels when the transmitter only knows this partial feedback. We prove that for cluster size $\frac{N}{L \sqrt{\log K}}$, when each user only feeds back the best SINR value at the center subcarrier of each cluster, the transmitter can perform opportunistic beamforming by constructing $M$ beams on each subcarrier and transmit to users with the best SINR values. This results in throughput scaling as with full CSI. Simulation results verify our analytical results and further show that larger cluster size $\left(\frac{N}{2 L}\right)$ and reduced feedback can be tolerated for a small throughput hit.

\section{APPENDIX A}

\section{Joint Probability Distribution ANALYsis}

This appendix evaluates the joint probability distribution (6). We substitute the closed form of the joint characteristic function (8) in the double integral of (6). We denote $j w_{p}$ with $s_{p}, j w_{q}$ with $s_{q}$, and perform contour integration where $s_{p}$ and $s_{q}$ go from $-\infty$ to $\infty$ on the complex axis (except for small region around the origin), then clock-wise towards $-\infty$. Fig. 6 illustrates the two contours $C_{s_{p}}$ and $C_{s_{q}}$. The double integral is shown in (A.1). We use Cauchy integral formula $\int_{C} \frac{f(z) d z}{z-z_{0}}=2 \pi j f\left(z_{0}\right)[18]$, which states that when a function $f$ is analytic on and within a simple contour $C$, we evaluate the integral by computing $f$ at points interior to $C$, in this case $z_{0}$. We start by evaluating the integral w.r.t. $s_{p}$. In $C_{s_{p}}$, we find two points $s_{p}=0$ and $s_{p}=\frac{L M s_{q}-1}{s_{q} M^{2}\left(L^{2}-f\right)-L M}{ }^{6}$. The integral at $s_{p}=0$ results in a term $\frac{1}{4}+\frac{1}{2} \frac{e^{\frac{-\gamma_{q}}{\rho L M}}}{\left[1+\gamma_{q}\right]^{M-1}}$ which cancels out with the third term in (6). Therefore the only point we consider is $s_{p}=\frac{L M s_{q}-1}{s_{q} M^{2}\left(L^{2}-f\right)-L M}$, and we compute its residue according to the residue theorem [18]. If we have a simple pole $z_{0}$, then $\operatorname{Res}\left(z_{0}\right)=\lim _{z \rightarrow z_{0}}\left(z-z_{0}\right) f(z)$. When pole $z_{0}$ is of order $m$, then $\operatorname{Res}\left(z_{0}\right)=\left.\frac{1}{(m-1) !} \frac{d^{m-1}}{d z^{m-1}}\left[\left(z-z_{0}\right)^{m} f(z)\right]\right|_{z=z_{0}}$.

Computing the residue at $s_{p}=\frac{L M s_{q}-1}{s_{q} M^{2}\left(L^{2}-f\right)-L M}$, the double integral (A.1) reduces to a single integral in terms of $s_{q}$ as shown in (A.2). To proceed, we find the roots of the polynomial (raised to $M-1$ ) in (A.2) in appendix B.

\section{APPENDIX B \\ ASYMPTOTIC ANALYSIS}

For the rest of the analysis, we assume the number of users $K$ is increasing, $M$ fixed, and the cluster size small enough so the correlation coefficient of $p$ and $q$ is $1-o\left(\frac{1}{\log K}\right)$ 7. In particular, the notation $O(\cdot)$ and $o(\cdot)$ are used when $K$ tends to infinity. We should remind the reader that the threshold for SINR at the center subcarrier $\gamma_{q}$ behaves like $\alpha_{q} \log K$, while the edge subcarrier's threshold $\gamma_{p}$ behaves like $\alpha_{p} \log K$, where $\alpha_{q}$ and $\alpha_{p}$ are constants and $\alpha_{q}>\alpha_{p}$.

\footnotetext{
${ }^{6}$ Since we assume that $\gamma_{q}>\gamma_{p}$.

${ }^{7}$ This implies that $\left(L^{2}-f\right) \rightarrow 0$, or equivalently that $p-q=\frac{N}{L \sqrt{\log K}}$.
} 


$$
\int_{C_{s_{q}}} \int_{C_{s_{p}}} \frac{-e^{\frac{-1}{\rho}\left(s_{p} \gamma_{p}+s_{q} \gamma_{q}\right)}}{4 \pi^{2} s_{p} s_{q}\left[1-\left(s_{p}+s_{q}\right) L M+s_{p} s_{q}\left(L^{2}-f\right) M^{2}\right]\left[1+\left(s_{p} \gamma_{p}+s_{q} \gamma_{q}\right) L M+s_{p} s_{q} \gamma_{p} \gamma_{q}\left(L^{2}-f\right) M^{2}\right]^{M-1}} d s_{p} d s_{q}
$$

$$
\begin{gathered}
\int_{C_{s_{q}}} \frac{-j}{2 \pi} \frac{e^{\frac{-\gamma_{q} s_{q}}{\rho}} e^{\frac{-\gamma_{p}}{\rho} \frac{\left(L M s_{q}-1\right)}{s_{q} M^{2}\left(L^{2}-f\right)-L M}}}{s_{q}\left(L M s_{q}-1\right)\left[1+L M\left(\gamma_{q} s_{q}+\gamma_{p}\left(\frac{L M s_{q}-1}{s_{q} M^{2}\left(L^{2}-f\right)-L M}\right)\right)+\left(\frac{L M s_{q}-1}{s_{q} M^{2}\left(L^{2}-f\right)-L M}\right) s_{q} \gamma_{p} \gamma_{q} M^{2}\left(L^{2}-f\right)\right]^{M-1}} d s_{q} \\
\int_{C_{s_{q}}} \frac{-j}{2 \pi} \frac{e^{\frac{-\gamma_{q} s_{q}}{\rho}}}{\left[1+\gamma_{p}\right]^{M-1} s_{q}\left[L M \frac{\left(\gamma_{q}-\gamma_{p}\right)}{1+\gamma_{p}} s_{q}+1+o(1)\right]^{M-1}\left(L M s_{q}-1\right)\left[\frac{-M\left(L^{2}-f\right) \gamma_{q}\left(1+\gamma_{p}\right)}{L\left(\gamma_{q}-\gamma_{p}\right)} s_{q}+1+o(1)\right]^{M-1}} d s_{q} \cdot
\end{gathered}
$$

Solving for the roots of the polynomial in (A.2) results in, Inside $C_{s_{q}}$ lies two points $s_{q}=\frac{1}{L M}$ which is a simple pole, and $s_{q}=\frac{L\left(\gamma_{q}-\gamma_{p}\right)}{M\left(L^{2}-f\right) \gamma_{q}\left(1+\gamma_{p}\right)}$ of order $M-1$. The residue at $s_{q}=0$ again gives a term $\frac{1}{2} \frac{e^{\frac{-\gamma_{p}}{\rho L M}}}{\left[1+\gamma_{p}\right]^{M-1}}$ which cancels out with the second term in (6). In order to use Cauchy integral formula [18], our function has to be analytic on and within the contour. Therefore we expand the second exponential in Eq. (B.1) as $e^{\frac{-\gamma_{p}}{\rho} \frac{\left(L M s_{q}-1\right)}{s_{q} M^{2}\left(L^{2}-f\right)-L M}}=\sum_{a=0}^{\infty} \frac{\left(\frac{-\gamma_{p}}{\rho}\right)^{a}}{a !}\left[\frac{L M s_{q}-1}{s_{q} M^{2}\left(L^{2}-f\right)-L M}\right]^{a}$. This summation results in 3 regions: $a \stackrel{2}{=} 0,0<a \leq M-1$ and $a \rightarrow \infty$. We cover each region in the following sections respectively. The result for the joint probability distribution is in (9).

\section{A. First Region $(a=0)$}

By performing partial fraction expansion on the two poles inside $C_{s_{q}}$, we obtain the equation at the top of the next page, where $g=\left.\frac{1}{\left[s_{q}-\frac{L\left(\gamma_{q}-\gamma_{p}\right)}{M\left(L^{2}-f\right) \gamma_{q}\left(1+\gamma_{p}\right)}\right]^{M-1}}\right|_{s_{q}=\frac{1}{L M}}=1+o(1)$ and $h_{i}=\left.\frac{1}{i !} \frac{d^{i}}{d s_{q}^{i}}\left[\frac{1}{s_{q}-\frac{1}{L M}}\right]\right|_{s_{q}=\frac{L\left(\gamma_{q}-\gamma_{p}\right)}{M\left(L^{2}-f\right) \gamma_{q}\left(1+\gamma_{p}\right)}}=o(1)$. Computing

the residue at each pole, we arrive at the following result for Eq. (B.1) when $a=0$,

$$
\frac{e^{-\frac{\gamma_{q}}{\rho L M}}}{\left(1+\gamma_{q}\right)^{M-1}}(1-o(1))^{M-1}+o\left(\gamma_{q}^{M-1} e^{\frac{-\gamma_{q}}{\rho} \frac{\left(\gamma_{q}-\gamma_{p}\right)}{\left(L^{2}-f\right) \gamma_{q}\left(1+\gamma_{p}\right)}}\right) \text {. }
$$

\section{B. Finite Region $(0<a \leq M-1)$}

We write the integral of Eq. (B.1) for one of these $M-1$ terms as shown in (B.3), which simplifies to (B.4). We calculate the residue at the only pole (of order $M-1) \frac{L\left(\gamma_{q}-\gamma_{p}\right)}{M\left(L^{2}-f\right) \gamma_{q}\left(1+\gamma_{p}\right)}$ as shown in the fourth equation from the top of the next page. For simplicity, we write $\frac{e^{\frac{-\gamma_{q}}{\rho} s_{q}}\left[1-s_{q} \frac{M\left(L^{2}-f\right)}{L}\right]^{M-a-1}\left[\frac{1}{L M}-s_{q}\right]^{a-1}}{s_{q}\left[L M \frac{\left(\gamma_{q}-\gamma_{p}\right)}{1+\gamma_{p}} s_{q}+1+o(1)\right]^{M-1}}=f\left(s_{q}\right)$. Eq.

becomes (B.5), which goes to zero as $\left(L^{2}-f\right) \rightarrow 0$, and does not contribute to (9).

\section{Infinite Region $(a \rightarrow \infty)$}

We write the integral of Eq. (B.1) as shown in (B.6). Looking at one of these terms and simplifying, we obtain (B.7). We observe two poles $s_{q}=\frac{L\left(\gamma_{q}-\gamma_{p}\right)}{M\left(L^{2}-f\right) \gamma_{q}\left(1+\gamma_{p}\right)}$ of order $M-1$ and $s_{q}=\frac{L}{M\left(L^{2}-f\right)}$ of order $(a-M+1)$. As we will soon see, the residues of these poles result in terms that go to 0 as $K$ increases and do not contribute to (9). First, we perform partial fraction expansion, as shown in (B.8). In the same manner used to find $h_{i}$ in appendix B.1, $x_{i}=\frac{(-1)^{a-M+1}(a-M+i) !}{i !(a-M) !}\left(L^{2}-f\right)^{a-M+i+1}$ and $y_{i}=\frac{(-1)^{i}(M+i-2) !}{i !(M-2) !}\left(L^{2}-f\right)^{M+i-1}$. We also simplify $\frac{e^{\frac{-\gamma_{q}}{\rho} s_{q}}\left[\frac{-1}{L M}+s_{q}\right]^{a-1}(-1)^{a-1}}{s_{q}\left[s_{q}+\frac{1+\gamma_{p}}{L M\left(\gamma_{q}-\gamma_{p}\right)}\right]^{M-1}}=H\left(s_{q}\right)$ and therefore the integral in Eq. (B.7) becomes (B.9), where (B.10) applies. Note that the first summation in Eq. (B.9) is bounded in $i$, and we observe a dominating value when $i=r_{1}$ and $r_{2}, r_{3}=0$. Therefore, $H^{(i)}\left(s_{q}\right)=$ $\frac{(-1)^{a-1}}{(M-2) !} e^{\frac{-\gamma_{q}}{\rho} s_{q}} \frac{(a-1) !}{(a-i-1) !} s_{q}^{a-(i+M+1)}$. Summing over $r_{2}$ and $r_{3}$, we obtain $\sum_{r_{2}, r_{3}}^{i-r_{1}} \frac{i !}{\left(i-r_{1}-r_{2}-r_{3}\right) !} \frac{1}{r_{3} !}\left(s_{q} \gamma_{q}\right)^{-r_{2}-r_{3}}(M+$ $\left.r_{3}-2\right) !=\frac{i !}{\left(i-r_{1}\right) !} i^{M-2}(1+o(1))$, then over $r_{1}$ gives $\mathrm{O}\left[\sum_{r_{1}=0}^{i} \frac{i ! i^{M-2}}{r_{1} !\left(a-r_{1}-1\right) !\left(i-r_{1}\right) !}\left(\frac{s_{q}}{\gamma_{q}}\right)^{r_{1}}(-1)^{r_{1}}\right]=$ $\mathrm{O}\left[\left(\frac{s_{q}}{\gamma_{q}}\right)^{i+1} \frac{i^{M}}{(a-1) !}\right]$. Therefore, $H^{(i)}\left(s_{q}\right)=$ $(-1)^{a-i-1} e^{\frac{-\gamma_{q}}{\rho} s_{q}} \frac{(a-1) !}{(a-i-1) !}\left(\frac{s_{q}}{\gamma_{q}}\right)^{i}$. We can now write the finite summation in $i$ as shown in the equation following (B.10) on the next page, which simplifies to

$$
\mathrm{O}\left[e^{\frac{-\gamma_{q}}{\rho\left(L^{2}-f\right)}} \sum_{a=M}^{\infty} M a^{M}\left(L^{2}-f\right)^{a}\right]_{\left(L^{2}-f\right)=o\left(\frac{1}{\gamma_{q}}\right)}^{\longrightarrow} 0
$$

The second summation in Eq. (B.9) can be written as shown in the last equation on the next page (for $a=M-1$ ), which results in

$$
\mathrm{O}\left[e^{\frac{-\gamma_{q}}{\rho\left(L^{2}-f\right)}} \sum_{a=M}^{\infty} \frac{a^{M}}{a !}\left[-\left(L^{2}-f\right)\right]^{a}\right] \quad \underset{\left(L^{2}-f\right)=o\left(\frac{1}{\gamma_{q}}\right)}{\longrightarrow} 0 .
$$




$$
\begin{aligned}
& \frac{1}{\left(s_{q}-\frac{1}{L M}\right)\left[s_{q}-\frac{L\left(\gamma_{q}-\gamma_{p}\right)}{M\left(L^{2}-f\right) \gamma_{q}\left(1+\gamma_{p}\right)}\right]^{M-1}}=\frac{g}{s_{q}-\frac{1}{L M}}+\sum_{i=0}^{M-2} \frac{h_{i}}{\left[s_{q}-\frac{L\left(\gamma_{q}-\gamma_{p}\right)}{M\left(L^{2}-f\right) \gamma_{q}\left(1+\gamma_{p}\right)}\right]^{M-1-i}} \\
& \mathrm{I}_{\mathrm{finite}}=\frac{e^{\frac{-\gamma_{q}}{\rho} s_{q}}\left[1-s_{q} \frac{M\left(L^{2}-f\right)}{L}\right]^{M-1} \frac{(-1)^{a}}{a !}\left(\frac{\gamma_{p}}{\rho}\right)^{a}\left[\frac{\frac{1}{L M}-s_{q}}{1-s_{q} \frac{M\left(L^{2}-f\right)}{L}}\right]^{a}}{-L M\left[1+\gamma_{p}\right]^{M-1} s_{q}\left(\frac{1}{L M}-s_{q}\right)\left[L M \frac{\gamma_{q}-\gamma_{p}}{1+\gamma_{p}} s_{q}+1+o(1)\right]^{M-1}\left[\frac{-M\left(L^{2}-f\right) \gamma_{q}\left(1+\gamma_{p}\right)}{L\left(\gamma_{q}-\gamma_{p}\right)} s_{q}+1+o(1)\right]^{M-1}} \\
& \mathrm{I}_{\text {finite }}=\frac{e^{\frac{-\gamma_{q}}{\rho} s_{q}}\left[1-s_{q} \frac{M\left(L^{2}-f\right)}{L}\right]^{M-a-1} \frac{(-1)^{a}}{a !}\left(\frac{\gamma_{p}}{\rho}\right)^{a}\left[\frac{1}{L M}-s_{q}\right]^{a-1}}{-L M s_{q}\left[1+\gamma_{p}\right]^{M-1}\left[L M \frac{\left(\gamma_{q}-\gamma_{p}\right)}{1+\gamma_{p}} s_{q}+1+o(1)\right]^{M-1}\left[\frac{-M\left(L^{2}-f\right) \gamma_{q}\left(1+\gamma_{p}\right)}{L\left(\gamma_{q}-\gamma_{p}\right)}\right]^{M-1}\left[s_{q}-\frac{L\left(\gamma_{q}-\gamma_{p}\right)}{M\left(L^{2}-f\right) \gamma_{q}\left(1+\gamma_{p}\right)}+o(1)\right]^{M-1}} . \\
& \left.\frac{1}{(M-2) !} \frac{d^{M-2}}{d s_{q}^{M-2}}\left(\frac{e^{\frac{-\gamma_{q}}{\rho} s_{q}}\left[1-s_{q} \frac{M\left(L^{2}-f\right)}{L}\right]^{M-a-1}\left[\frac{1}{L M}-s_{q}\right]^{a-1}}{s_{q}\left[L M \frac{\left(\gamma_{q}-\gamma_{p}\right)}{1+\gamma_{p}} s_{q}+1+o(1)\right]^{M-1}}\right)\right|_{s_{q}=\frac{L\left(\gamma_{q}-\gamma_{p}\right)}{M\left(L^{2}-f\right) \gamma_{q}\left(1+\gamma_{p}\right)}} . \\
& \mathrm{I}_{\text {finite }}=\left.\frac{\left(-\gamma_{p}\right)^{a}}{a !(\rho)^{a}} \frac{1}{(M-2) !(-L M)\left[1+\gamma_{p}\right]^{M-1}} f_{s_{q}}^{M-2}\right|_{s_{q} \rightarrow \infty}=\mathrm{O}\left[\frac{\left(-\gamma_{p}\right)^{a}}{\left[1+\gamma_{p}\right]^{M-1}} e^{\frac{-\gamma_{q}\left(\gamma_{q}-\gamma_{p}\right)}{\rho\left(L^{2}-f\right) \gamma_{q}\left(1+\gamma_{p}\right)}}\left(\frac{-1}{\left(L^{2}-f\right)}\right)^{M-2}\right] \\
& \mathrm{I}_{\text {infinite }}=\frac{e^{\frac{-\gamma_{q}}{\rho} s_{q}}\left[1-s_{q} \frac{M\left(L^{2}-f\right)}{L}\right]^{M-1} \sum_{a=M}^{\infty} \frac{(-1)^{a}}{a !}\left(\frac{\gamma_{p}}{\rho}\right)^{a}\left[\frac{\frac{1}{L M}-s_{q}}{1-\frac{M\left(L^{2}-f\right)}{L} s_{q}}\right]^{a}}{-L M\left[1+\gamma_{p}\right]^{M-1}\left[-M^{2}\left(L^{2}-f\right) \gamma_{q}\right]^{M-1} s_{q}\left(\frac{1}{L M}-s_{q}\right)\left[s_{q}+\frac{1+\gamma_{p}}{L M\left(\gamma_{q}-\gamma_{p}\right)}\right]^{M-1}\left[s_{q}-\frac{L\left(\gamma_{q}-\gamma_{p}\right)}{M\left(L^{2}-f\right) \gamma_{q}\left(1+\gamma_{p}\right)}\right]^{M-1}} . \\
& \mathrm{I}_{\text {infinite }}=\frac{-\left(\frac{-\gamma_{p}}{\rho}\right)^{a}}{a ! L M\left[1+\gamma_{p}\right]^{M-1} \gamma_{q}^{M-1}\left(L^{2}-f\right)^{a}} \frac{e^{\frac{-\gamma_{q}}{\rho} s_{q}}\left[\frac{1}{L M}-s_{q}\right]^{a-1}}{s_{q}\left[s_{q}+\frac{1+\gamma_{p}}{L M\left(\gamma_{q}-\gamma_{p}\right)}\right]^{M-1}} \frac{1}{\left[s_{q}-\frac{L}{M\left(L^{2}-f\right)}\right]^{a-M+1}\left[s_{q}-\frac{L\left(\gamma_{q}-\gamma_{p}\right)}{M\left(L^{2}-f\right) \gamma_{q}\left(1+\gamma_{p}\right)}\right]^{M-1}} . \\
& \frac{1}{\left[s_{q}-\frac{L\left(\gamma_{q}-\gamma_{p}\right)}{M\left(L^{2}-f\right) \gamma_{q}\left(1+\gamma_{p}\right)}\right]^{M-1}\left[s_{q}-\frac{L}{M\left(L^{2}-f\right)}\right]^{a-M+1}}=\sum_{i=1}^{M-1} \frac{x_{i}}{\left[s_{q}-\frac{L\left(\gamma_{q}-\gamma_{p}\right)}{M\left(L^{2}-f\right) \gamma_{q}\left(1+\gamma_{p}\right)}\right]^{i}}+\sum_{i=1}^{a-M+1} \frac{y_{i}}{\left[s_{q}-\frac{L}{M\left(L^{2}-f\right)}\right]^{i}} . \\
& \mathrm{I}_{\text {infinite }}=\frac{-\left(\frac{-\gamma_{p}}{\rho}\right)^{a}}{a ! L M\left[1+\gamma_{p}\right]^{M-1} \gamma_{q}^{M-1}\left(L^{2}-f\right)^{a}}\left[\sum_{i=1}^{M-1} \frac{x_{i}}{i !} H^{(i)}\left(\frac{L\left(\gamma_{q}-\gamma_{p}\right)}{M\left(L^{2}-f\right) \gamma_{q}\left(1+\gamma_{p}\right)}\right)+\sum_{i=1}^{a-M+1} \frac{y_{i}}{i !} H^{(i)}\left(\frac{L}{M\left(L^{2}-f\right)}\right)\right] \\
& H^{(i)}\left(s_{q}\right)=(-1)^{a-i-1} \frac{(a-1) !}{(M-2) !} e^{\frac{-\gamma_{q}}{\rho} s_{q}} \sum_{r_{1}, r_{2}, r_{3}}^{i} \frac{(-1)^{r_{1}} i !}{r_{1} ! r_{3} !\left(i-r_{1}-r_{2}-r_{3}\right) !} \frac{\left(M+r_{3}-2\right) !}{\left(a-r_{1}-1\right) !} \gamma_{q}^{i-r_{1}}\left(s_{q} \gamma_{q}\right)^{-r_{2}-r_{3}} s_{q}^{a-r_{1}-M-1} \\
& \mathrm{O}\left[\sum_{a=M}^{\infty} \sum_{i=1}^{M-1} \frac{(a-i-1) !}{i !(M-i-1) !(a-M+1) !}\left(L^{2}-f\right)^{a-M+i}(-1)^{a-M+i}(-1)^{a-1} e^{\frac{-\gamma_{q}}{\rho\left(L^{2}-f\right)}}\left(L^{2}-f\right)^{1+M-i}\right] \\
& \mathrm{O}\left[(a-1) !(-1)^{a}\left(L^{2}-f\right)^{a} e^{\frac{-\gamma_{q}}{\rho\left(L^{2}-f\right)}} \sum_{i=1}^{a-M+1} \frac{1}{i !(a-M-i+1) !}\left(\frac{1}{\left(L^{2}-f\right) \gamma_{q}}\right)^{i}\right]
\end{aligned}
$$




\section{REFERENCES}

[1] M. Sharif and B. Hassibi, "On the capacity of MIMO broadcast channel with partial side information," IEEE Trans. Inform. Theory, vol. 51, Feb. 2005.

[2] G. Caire and S. Shamai, "On the achievable throughput of a multiantenna Gaussian broadcast channel," IEEE Trans. Inform., vol. 49, no. 7, pp. 1691-1706, July 2003.

[3] P. Viswanath and D. N. Tse, "Sum capacity of the vector Gaussian broadcast channel and downlink-uplink duality," IEEE Trans. Inform., vol. 49, no. 8, pp. 1912-1921, Aug. 2003.

[4] S. Vishwanath, N. Jindal, and A. Goldsmith, "Duality, achievable rates and sum rate capacity of Gaussian MIMO broadcast channel," IEEE Trans. Inform., vol. 49, no. 10, pp. 2658-2668, 2003.

[5] H. Weingarten, Y. Steinberg, and S. Shamai, "The capacity region of the gaussian MIMO broadcast channel," in Proc. IEEE ISIT, 2004.

[6] M. Sharif and B. Hassibi, "Scaling laws of sum rate using time-sharing, DPC, and beamforming for MIMO broadcast channels," in Proc. IEEE ISIT, 2004.

[7] P. Svedman, S. Wilson, L. Cimini, and B. Ottersten, "A simplified opportunistic feedback and scheduling scheme for OFDM," in Proc. IEEE Veh. Tech. Conference, May 2004.

[8] P. Svedman, L. J. Cimini, M. Bengtsson, S. K. Wilson, and B. Ottersten, "Exploiting temporal channel correlation in opportunistic SD-OFDMA," in Proc. IEEE International Conference on Communications, June 2006.

[9] D. Gesbert and M. Alouini, "How much feedback is multi-user diversity really worth?" in Proc. IEEE International Conference on Communications, June 2004.

[10] S. Sanayei, A. Nosratinia, and N. Aldhahir, "Opportunistic dynamic subchannel allocation in multiuser OFDM networks with limited feedback," in Proc. IEEE Inform. Theory Workshop, Oct. 2004.

[11] S. Sanayei and A. Nosratinia, "Exploiting multiuser diversity with only 1-bit feedback," in Proc. IEEE Wireless Communications and Networking Conference, vol. 2, Mar. 2005.

[12] L. Toufik and R. Knopp, "Multiuser channel allocation algorithms achieving hard fairness," in Proc. IEEE Global Communications Conference, vol. 1, Dec. 2004.

[13] D. Gesbert and M.-S. Alouini, "Selective multi-user diversity," in Proc. IEEE International Symp. on Signal Processing and Information Technology, Dec. 2003.

[14] S. Colieri, M. Ergen, A. Puri, and A. Bahai, "A study of channel estimation in OFDM systems," in Proc. IEEE Vehicular Technology Conference, vol. 2, Sept. 2002.

[15] J. Wang and B. Daneshrad, "Performance of linear interpolation-based MIMO detection for MIMO-OFDM systems," in Proc. IEEE Wireless Communications and Networkiing Conference, vol. 2, Mar. 2004.

[16] D. Tse and P. Viswanath, Fundamentals of Wireless Communication. Cambridge University Press, 2005.

[17] J. Proakis, Digital Communications. New York: McGraw-Hill Publishing Co., 2001.

[18] R. Churchill and J. Brown, Complex Variables and Applications. New York: McGraw-Hill Publishing Co., 1990.

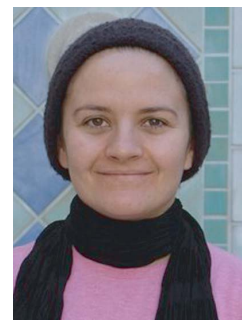

Maralle Jamal Fakhereddin received the B.S. and M.S. degrees in electrical and computer engineering from Drexel University, Philadelphia, PA, in 2000, and 2003, respectively. From June 2004 to June 2006, she was a Research Intern at California Institute of Technology, Pasadena, CA. She is currently with VMware, Palo Alto, CA. Her research interests are in the areas of wireless communications and information theory.

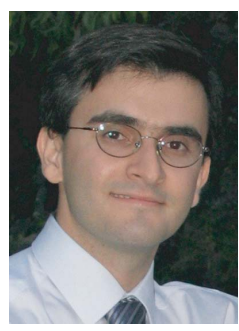

Masoud Sharif received his Ph.D. in Electrical Engineering (2005) from California Institute of Technology. In 2005, he was a post-doctoral scholar in the EE department at Caltech. Since January 2006, he has been an assistant Professor at Boston University. Dr. Sharif was awarded the C.H.Wilts Prize in 2006 for best doctoral thesis in Electrical Engineering at Caltech. He is a member of the Center for Information and Systems Engineering at Boston University. His research interests include adhoc and sensor networks, multiple-user multipleantenna communication channels, crosslayer design for wireless networks, and multi-user information theory. His recent research has focused on collaborative communication scheme in ad-hoc and sensor networks and the capacity of multiple antenna broadcast channels.

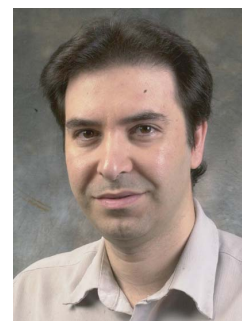

Babak Hassibi was born in Tehran, Iran, in 1967. He received the B.S. degree from the University of Tehran in 1989, and the M.S. and Ph.D. degrees from Stanford University in 1993 and 1996, respectively, all in electrical engineering. From October 1996 to October 1998 he was a research associate at the Information Systems Laboratory, Stanford University, and from November 1998 to December 2000 he was a Member of the Technical Staff in the Mathematical Sciences Research Center at Bell Laboratories, Murray Hill, NJ. Since January 2001 he has been with the department of electrical engineering at the California Institute of Technology, Pasadena, CA., where he is currently professor and executive officer. He has also held short-tem appointments at Ricoh California Research Center, the Indian Institute of Science, and Linkoping University, Sweden. His research interests include wireless communications, robust estimation and control, adaptive signal processing and linear algebra. $\mathrm{He}$ is the coauthor of the books Indefinite Quadratic Estimation and Control: A Unified Approach to H2 and H1 Theories (New York: SIAM, 1999), and Linear Estimation (Englewood Cliffs, NJ: Prentice Hall, 2000). He is a recipient of an Alborz Foundation Fellowship, the 1999 O. Hugo Schuck best paper award of the American Automatic Control Council, the 2002 National Science Foundation Career Award, the 2002 Okawa Foundation Research Grant for Information and Telecommunications, the 2003 David and Lucille Packard Fellowship for Science and Engineering and the 2003 Presidential Early Career Award for Scientists and Engineers (PECASE). $\mathrm{He}$ has been a Guest Editor for the IEEE TRANSACTIONS ON INFORMATION THEORY special issue on space-time transmission, reception, coding and signal processing, was an Associate Editor for Communications of the IEEE TRANSACTIONS ON INFORMATION THEORY during 2004-2006, and is currently an Editor for the journal FOUNDATIONS AND TRENDS IN INFORMATION AND COMMUNICATION. 